\title{
CFD ANALYSES OF COOLANT CHANNEL FLOWFIELDS
}

\author{
Jennifer A. Yagley ${ }^{\dagger}$, Jinzhang Feng ${ }^{\ddagger}$, and Charles L.Merkle ${ }^{\S}$ \\ Propulsion Engineering Research Center \\ Department of Mechanical Engineering \\ The Pennsylvania State University \\ University Park, PA 16802
}

\section{SUMMARY}

The flowfield characteristics in rocket engine coolant channels are analyzed by means of a numerical model. The channels are characterized by large length to diameter ratios, high Reynolds numbers, and asymmetrical heating. At representative flow conditions, the channel length is approximately twice the hydraulic entrance length so that fully developed conditions would be reached for a constant property fluid. For the supercritical hydrogen that is used as the coolant, the strong property variations create significant secondary flows in the cross-plane which have a major influence on the flow and the resulting heat transfer. Comparison of constant and variable property solutions show substantial differences. In addition, the property variations prevent fully developed flow. The density variation accelerates the fluid in the channels increasing the pressure drop without an accompanying increase in heat flux. Analyses of the inlet configuration suggest that side entry from a manifold can affect the development of the velocity profile because of vortices generated as the flow enters the channel.Current work is focused on studying the effects of channel bifurcation on the flow field and the heat transfer characteristics.

\section{TECHNICAL DISCUSSION}

Regenerative cooling is normally used in rocket engine combustors to protect the walls from the severe thermal environment of the combustion process. In most cases, the coolant passages are rectangular in shape and run the length of the combustor with the coolant entering at the supersonic end, passing through the throat region, and exiting near the injector face. In cryogenic engines, the coolant is generally liquid hydrogen, and the elevated pressures are sufficient to ensure that the fluid remains supercritical over the entire length of the cooling passage.

Although the design of these coolant passages is critical to the operation and life of the engine, their heat transfer characteristics are only poorly understood. To maximize our understanding of the fluid dynamics and the associated heat transfer characteristics of the coolant passages, the limiting case of a straight rectangular channel (which is currently being tested at NASA LeRC) is studied. Even with straight geometries, the flowfield inside the tubes remains quite complex because of the asymmetrical heating, the fin effect, the presence of a supercritical coolant with its characteristically strong property variations and the high Reynolds numbers. Because of the complex three-dimensional flow patterns in these coolant passages, it is anticipated that an optimum channel aspect ratio exists, and a validated CFD code is an important tool in assessing where this optimum occurs under different conditions.

At supercritical conditions all fluid properties (density, viscosity, thermal conductivity and specific heat) become strong functions of both pressure and temperature [1]. These variations are particularly strong near the critical

$\dagger$ Graduate Research Assistant

$\ddagger$ Research Associate

$\S$ Distinguished Alumni Professor 
point. Temperature dependence is especially significant in the present problem because of the strong temperature gradients near the hot wall of the coolant passage.

In the presence of property variations, the Navier-Stokes equations and the energy equation are coupled and must be solved simultaneously. The corresponding system of governing equations is:

$$
\Gamma \frac{\partial Q}{\partial \tau}+\frac{\partial}{\partial \xi}(E-E v)+\frac{\partial}{\partial \eta}(F-F v)+\frac{\partial}{\partial \zeta}(G-G v)=0
$$

$\mathrm{Q}=(\mathrm{P}, \mathrm{u}, \mathrm{v}, \mathrm{w}, \mathrm{T})^{\mathrm{T}}$ is the vector of dependent variables; $(\xi, \eta, \zeta)$ represents a general non-orthogonal coordinate system; $\Gamma$ is a pre-conditioning matrix; $E, F$, and $G$ are the conservative flux vectors while $E_{v} F_{v}$ and $G_{v}$ are the viscous flux vectors [2]. The equation set is completed by a tabular equation of state relating the density to the local temperature and pressure. Additionally, auxiliary equations for the pressure and temperature dependence of the viscosity, thermal conductivity and specific heat are also specified. The Baldwin-Lomax algebraic turbulence model [3] is used to accommodate turbulent flow. The turbulent Prandtl number is chosen as 0.9 throughout the calculation.

Although our interest is in steady flow, the equations of motion are written in unsteady form for computational purposes and are solved by a four stage Runge-Kutta explicit scheme [4]. All derivatives of the Navier-Stokes equations are represented by centered differences. Local time stepping is used to achieve maximum convergence and a small amount of fourth order artificial dissipation is added to prevent odd-even splitting. Since the time derivatives disappear in the steady state, some license may be taken with them to counter the stiffness caused by the low Mach numbers in the supercritical fluid. This stiffness is circumvented using an artificial compressibility expression $[5,6]$. During the process of convergence, all the time derivatives approach zero and the numerical solution satisfies the proper steady state Navier-Stokes equations.

As boundary conditions for the present calculations, we specify the velocity and temperature profiles at the inlet, and uniform pressure at the exit plane. The remaining flow variables at the inlet and outlet are computed from the Riemann variables determined from the method of characteristics [4]. The velocity boundary conditions on the passage wall are the traditional no-slip conditions. These are augmented by enforcing the normal momentum equation to obtain the wall pressure.

Heat transfer conditions representative of those in combustor coolant passages are simulated by selecting appropriate Dirichlet (temperature) boundary conditions around the periphery of the duct. Uniform temperature conditions are assigned on both the inner (combustor) and outer (ambient) sides of the passage while a linear distribution of temperature is used on the side walls to simulate the fin effect. The inlet temperature is specified as $40 \mathrm{~K}$. Future plans are to couple the present fluids solution with a heat conduction formulation for the combustor walls to obtain the coupled solution.

The straight duct study was conducted to give an understanding of the fundamental physical effects of variable properties, turbulence modeling, high length to diameter ratios and inlet conditions. The next step in the study is to investigate the effects of channel bifurcation on the flow and temperature fields. A representative geometry is shown in Figure 1.

\section{RESULTS}

The geometry and flow conditions for this study were chosen to parallel an experimental study currently being conducted at NASA LeRC [7]. The length of the experimental channel is $137 \mathrm{~mm}$ which corresponds to 112 hydraulic diameters. The Reynolds number based on the inlet conditions and hydraulic diameter is 500,000 . For the current study we also studied channels of shorter lengths and flow at the lower Reynolds number of 10,000. Computations have also been completed for both constant and variable (supercritical hydrogen) property flows. 
One of the important issues in the straight tube studies is the effect of variable properties on the pressure drop and heat transfer characteristics of the channels. The property variation of super-critical hydrogen significantly alters the flow field characteristics and it is important to assess its effect on the channel cooling. To evaluate the nature of these differences a comparison of the flowfields based upon constant and variable properties was conducted. This comparison was made at a Reynolds number of 10,000.

The mid-plane, streamwise direction contour plots of the pressure, the axial velocity and the temperature variations are shown in Figure 2. The constant property results are shown on the right while the variable property results are shown on the left. The cross-stream direction has been stretched by a factor of five in theses figures. As can be seen, the variable properties cause the velocity profiles to be very non-uniform across the tube, and in addition, lead to an acceleration by a factor of about two. The higher flow speeds cause a lesser, but noticeable effect on the temperature.

A comparison of the flow fields for the two different Reynolds numbers 10,000 and 500,000 shows the viscous and thermal boundary layers were significantly thinner at the higher Reynolds number. Despite the fact that the thermal effect are limited to a small portion of the flowfield there is still an acceleration of the flow due to the density variations although not as significantly as in the lower Reynolds number.

Another issue studied was the effect of inlet velocity profiles on the downstream fluid dynamics and heat transfer. The inlet conditions studied were slug flow, fully developed velocity profiles and entry through a 90 degree bend (constant property). The first two represents a bounding study between two extremes in inlet profiles. The last condition represents an approximation to the type of entry that is used in the experimental configuration. A comparison of fully developed velocity profile with the slug flow velocity profile shows that the inlet condition has a measurable effect on the velocity contours in the exit plane (Fig. 3). The contours in the exit plane are qualitatively similar, but the results for the slug flow inlet show a peak velocity of $4.0 \mathrm{~m} / \mathrm{s}$ in the exit plane whereas those for the fully developed inlet show a peak of $3.3 \mathrm{~m} / \mathrm{s}$. The corresponding pressure drop for the slug flow inlet is about 15 percent larger than that for the fully developed inlet profile. By contrast, the fluid temperatures at the exit plane for both cases are about the same. Both calculations show that the fluid has been heated to the point where the minimum temperature in the exit plane is $45 \mathrm{~K}$, although more of the fluid appears to have been heated in the slug flow inlet case. In agreement with this latter observation, computation of the heat flux through the walls shows that the heat addition is larger (by about 15 percent) for the slug flow inlet case. These differences between the two calculations are not major, but they do suggest that care should be taken in defining the inlet conditions to be sure to obtain maximum accuracy in the coolant channel predictions.

The results for the second inlet condition, the inlet with a 90 degree bend are presented in Figure 4 . The velocity contours are quite similar in magnitude, although the profiles for the 90 degree bend inlet are slightly asymmetric. Similar comparison of the temperature profiles shows the presence of the bend in the inlet increases the minimum temperature from $90 \mathrm{~K}$ to $110 \mathrm{~K}$, suggesting that the vortex created by the turn at the inlet has some effect on the heat flux.

Comparisons of the streamwise development of the various flow characteristics are given on Figs. 5 and 1. The pressure drop predictions for the two cases is nearly identical (not counting the pressure drop across the inlet bend itself), suggesting that the strength of the vortices developed by the bend in the inlet was not sufficient to affect the downstream pressure gradient. The velocity profile in the bent inlet calculation shows a dramatic thickening of the boundary layer downstream of the inside portion of the curved inlet. This arises from a flow separation near the bend. The presence of flow separation and the vortex generated by the curved inlet suggests that the type of connection between the manifold and the coolant channels can have an effect on the downstream heat transfer. 


\section{REFERENCES}

[1] McCarty, R.D., "Hydrogen Technological Survey-Thermophysical Properties", NASA SP-3089, 1975, Washington D.C.

[2] Yagley,J.A., Feng, J. and Merkle, C.L., "CFD Analyses of Coolant Channel Flowfields", AIAA/S AE/ASME/ ASEE 29th Joint Propulsion Conference, AIAA-93-1830, June 1993, Monterey, CA.

[3] Baldwin, B.S. and Lomax H., "Thin Layer Approximation and Algebraic Model for Separated Turbulent Flows", AIAA 16th Aerospace Sciences Meeting, AIAA Paper 78-257, January 1978, Huntsville, AL.

[4] Merkle, C.L. and Tsai, Y.L. Peter, "Application of Runge-Kutta Schemes to Incompressible Flows", AIAA-86-0553, AIAA 24th Aerospace Sciences Meeting, January 1986, Reno, NV.

[5] Merkle,C.L. and Athavale, M., "Time-accurate Unsteady Incompressible Flow Algorithms based on Artificial Compressibility", AIAA 8th Computational Fluid Dynamics Conference, AIAA Paper 87-1137, June 1987, Honolulu, HA.

[6] Choi, Y.-H. and Merkle, C.L., "The Application of Preconditioning in Viscous Flows", Journal of Computation Physics, Vol 105, No2, April 1993.

[7] Carlile, J.A. and Quentmeyer, R.J., "An Experimental Investigation of High-Aspect-Ratio Cooling Passages", AIAA-92-3154, AIAA/SAE/ASME/ASEE 28th Joint Propulsion Conference, July 1992, Nashville, TN.
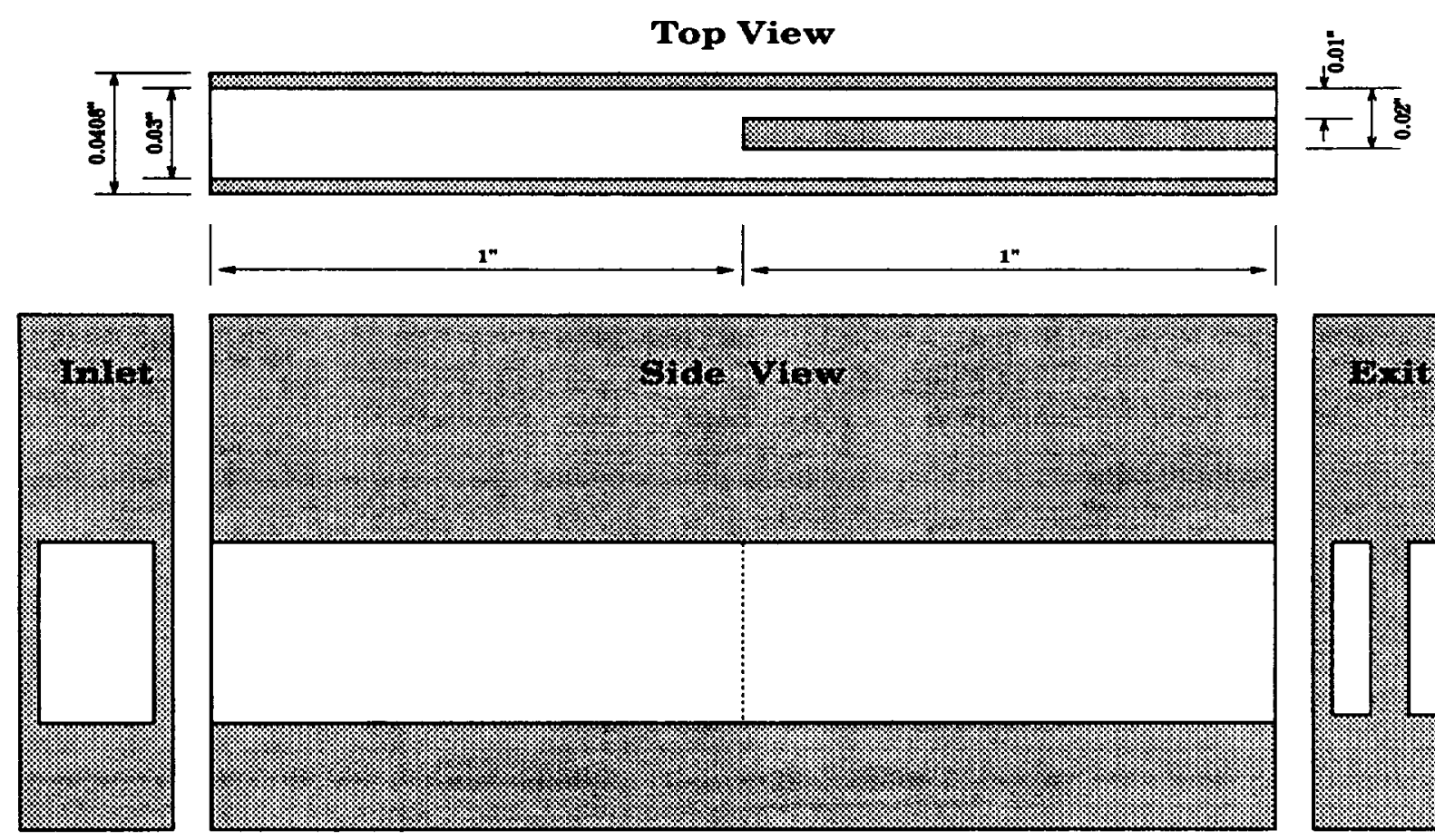

Figure 1: Representative sketch of channel bifurcation geometry. 

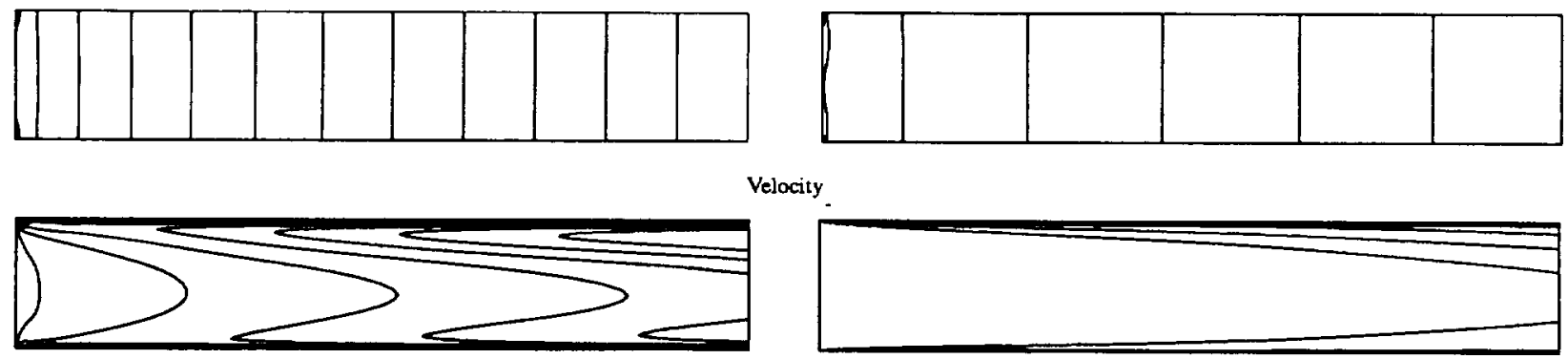

Temperature
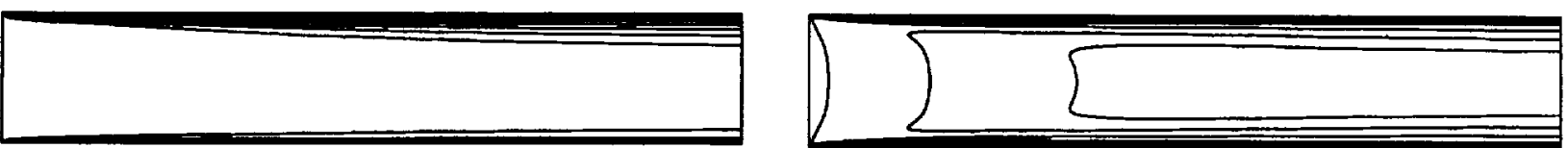

Figure 2: Steamwise contours of the pressure, velocity, and temperature on the centerplane for an $L / D_{h}$ of 28 . Variable Properties: $P_{\text {inc }}=20 ., P_{\max }=220 ; u_{\text {inc }}=0.5, u_{\max }=4.0 ; T_{\text {inc }}=70, T_{\max }=600$ (top wall). Constant Properties: $\mathrm{P}_{\text {inc }}=6 ., \mathrm{P}_{\max }=42 . ; \mathrm{u}_{\text {inc }}=0.17, \mathrm{u}_{\max }=1.4 ; \mathrm{T}_{\text {inc }}=70, \mathrm{~T}_{\max }=600$ (top wall).
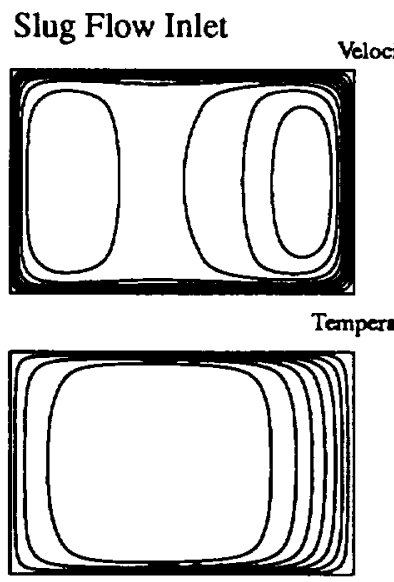

Temperature

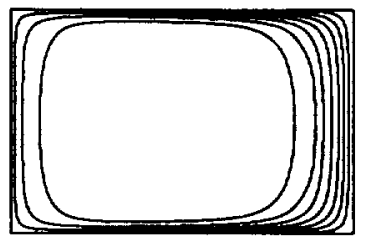

Figure 3: Contour of axial velocity and temperature at the exit plane $\left(L / D_{h}=28\right)$ for slug flow and fully developed inlet with variable properties. Slug: $u_{\text {inc }}=0.4, u_{\max }$ $=4.0, \mathrm{~T}_{\mathrm{inc}}=70, \mathrm{~T}_{\min }=45$. Fully Developed: $\mathrm{u}_{\mathrm{inc}}=0.4$, $\mathrm{u}_{\max }=3.3 ; \mathrm{T}_{\mathrm{inc}}=70, \mathrm{~T}_{\max }=600$ (right wall).
Slug Flow Inlet

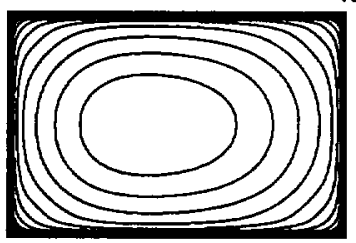
Velocity

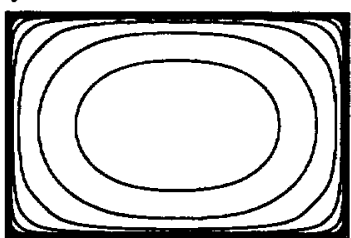

Temperature
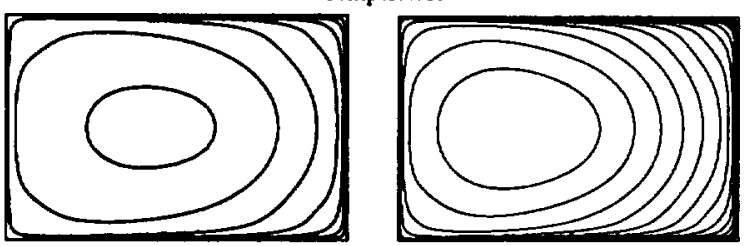

Figure 4: Contour of axial velocity and temperature in the exit plane $\left(L / D_{h}=28\right)$ for slug flow and bend entrance inlet with constant properties. Slug: $u_{i n c}=0.4, u_{\max }=4.0$, $\mathrm{T}_{\text {inc }}=70, \mathrm{~T}_{\min }=45$. Bend Inlet: $\mathrm{u}_{\mathrm{inc}}=0.4, \mathrm{u}_{\max }=3.3$; $T_{\text {inc }}=70, T_{\max }=600$ (right wall).

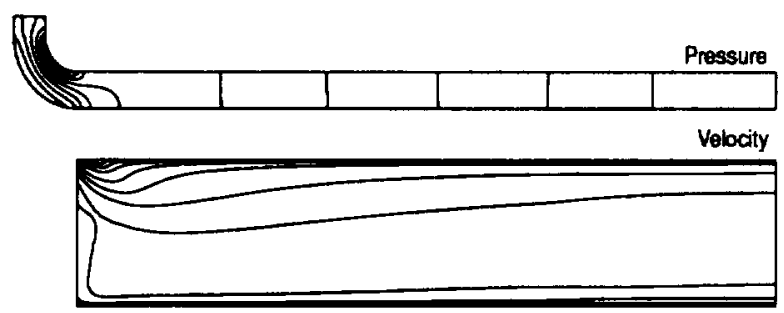

Temperature

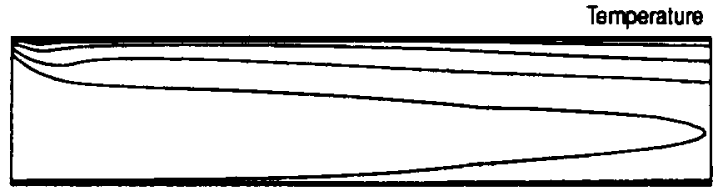

Figure 5: Effects of bend on flowfield (constant properties). There are 28 hydraulic diameters after the bend. $P_{i n c}=0.5, P_{\max }=5 ; u_{i n c}=0.1, u_{\min }=-0.1, u_{\max }=1.5 ; T_{i n c}=70, T_{\max }=600$ (at bottom wall), $\mathrm{Tmin}=40$ (at inlet). 